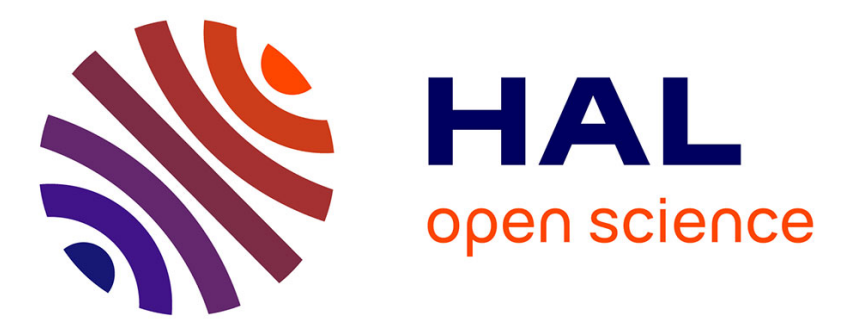

\title{
Comment un livre change : Cities in Evolution et les usages de Patrick Geddes (1912-1972) \\ Pierre Chabard
}

\section{To cite this version:}

Pierre Chabard. Comment un livre change : Cities in Evolution et les usages de Patrick Geddes (1912-1972). Genèses. Sciences sociales et histoire, 2005, 60 (3), pp.76. 10.3917/gen.060.0076 . hal-02513193

\section{HAL Id: hal-02513193 https://hal.science/hal-02513193}

Submitted on 23 Mar 2020

HAL is a multi-disciplinary open access archive for the deposit and dissemination of scientific research documents, whether they are published or not. The documents may come from teaching and research institutions in France or abroad, or from public or private research centers.
L'archive ouverte pluridisciplinaire HAL, est destinée au dépôt et à la diffusion de documents scientifiques de niveau recherche, publiés ou non, émanant des établissements d'enseignement et de recherche français ou étrangers, des laboratoires publics ou privés. 


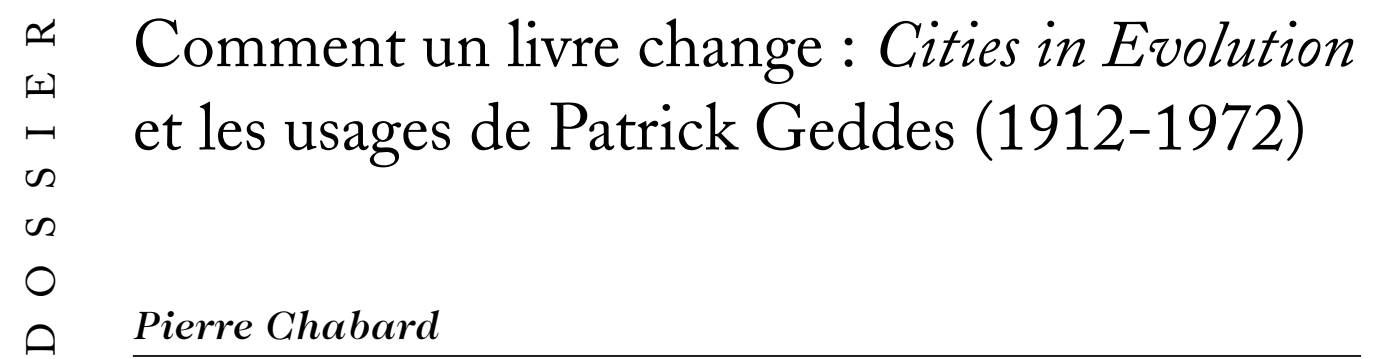

PP. $123-178$

"Dans ce livre, Patrick Geddes a déployé une compréhension globale de la scène urbaine et a montré que le town planning était plus qu'une simple expertise technologique ou esthétique. Il a instauré une tradition du planning qui n'a cessé d'être redécouverte dans les soixante dernières années: celle d'une synthèse à l'àge de la spécialisation, apportant harmonie et compréhension au complexe physique, économique et social qu'est la ville» (Cherry 1974 : 51-52).

Cet article rend compte d'une enquête historiographique dont l'objet est un livre, et son devenir durant le demi-siècle qui a suivi sa parution. À rebours du genre littéraire de la «fortune critique», il ne s'agira nullement de retracer la postérité de cet ouvrage - reconnu aujourd'hui comme un «classique»- de mesurer son aire d'influence ou de rayonnement, telle des cercles concentriques déployés autour d'un point d'origine indiscutable. Le regard sera ici fondamentalement renversé et décentré. Ce sont d'abord l'identité, la consistance et la permanence mêmes de cet objet éditorial, resitué dans ses successifs contextes d'apparition, qui seront questionnées. Bien au-delà de la question de la «réception», ce sont les usages, les manipulations - dans tous les sens du terme - dont il a fait l'objet qui seront ensuite examinés comme autant de moments créateurs du livre. Car le processus qui fige un livre dans le statut de classique est aussi paradoxalement celui qui le précipite vers l'instabilité et la métamorphose éditoriales. Réédition intégrale ou abrégée, traduite ou armée d'un jeu d'annexes, par fragments dans une anthologie ou même comme longue citation dans un autre texte, toutes ces opérations changent le livre, filtrent son contenu, orientent sa lecture, amendent sa forme. Il ne s'agit pas de nier les intentions particulières de celui qui, d'abord, l'a écrit mais d'envisager avec autant d'attention celles, souvent collectives, de ceux qui s'en sont saisis et en ont fait usage; et de comprendre pourquoi et surtout comment on lit, après quatre-vingt-dix ans, un livre de 1915. 

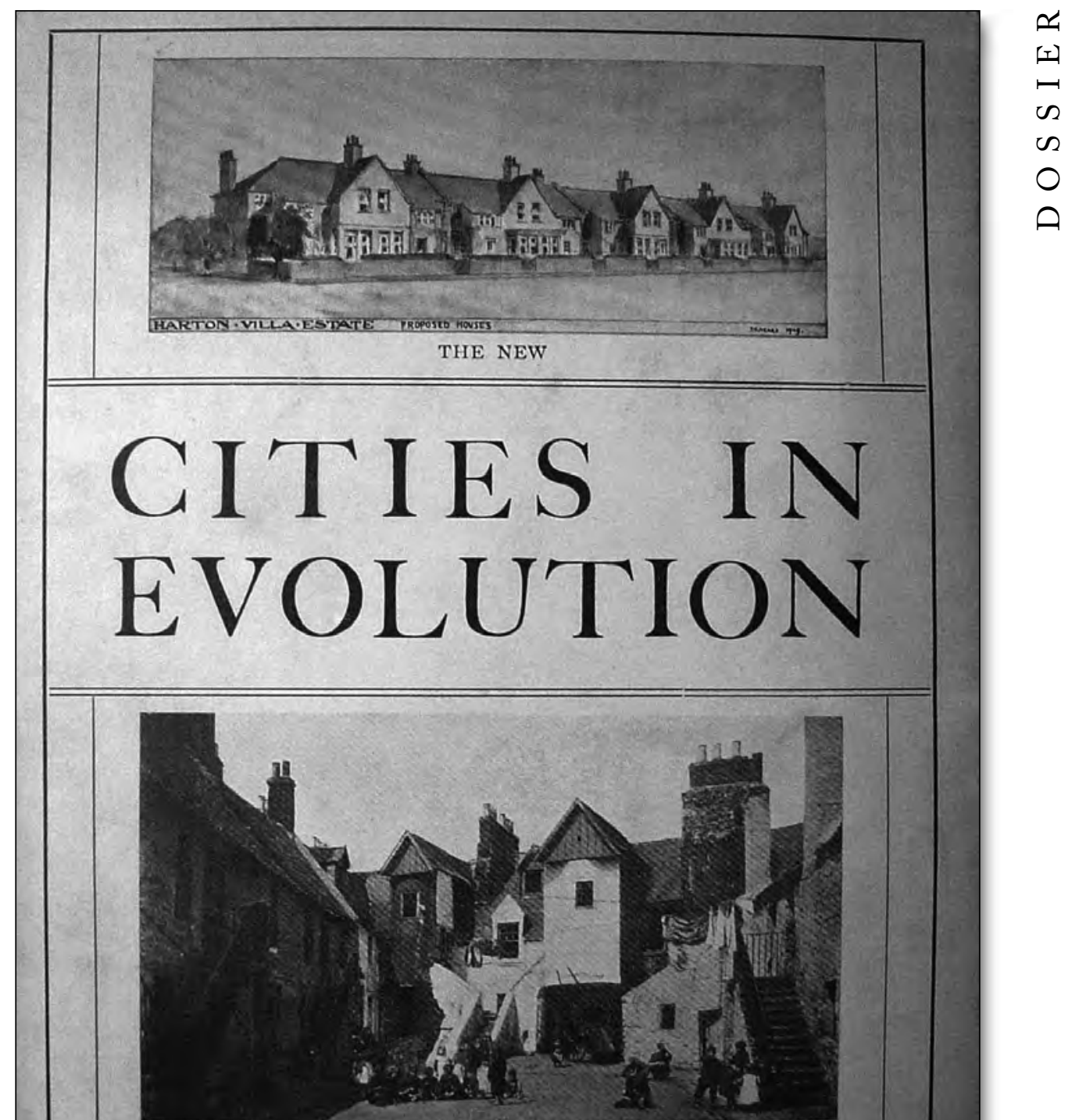

THE OLD

PATRICK DDES

WILLIAMS

${ }_{14}$ HENRIETTA

Couverture de l'édition originale de Cities in Evolution (Londres, Williams \& Norgate, 1915) 
또

띠

$\mapsto$

u

u

0

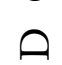

\section{Un illisible classique}

Cities in Evolution: an Introduction to the town planning Movement and to the Study of Civics (Geddes 1915) compte incontestablement parmi les classiques de la littérature urbaine. "Repère dans l'histoire du town planning" (Cherry 1974 : 51), «jalon dans le développement de la pensée sociale de ce siècle» (Clavel 1971 : vii), cet ouvrage «reste une des sources les plus fondamentales pour le town-planner, le sociologue, l'historien, pour tous les étudiants et pour le citoyen responsable» (Stalley 1972 : 109). Plus que tout autre écrit de Patrick Geddes, ce livre jouit d'une large reconnaissance dans les domaines du savoir et de l'action dont la ville constitue un des objets: principalement l'urbanisme et son histoire, la sociologie urbaine, la géographie mais également l'écologie ou l'environnementalisme. L'importance généalogique que ces différentes traditions savantes accordent au livre se traduit dans les usages éditoriaux. À titre d'exemple, Cities in Evolution figure aujourd'hui, sous trois formes différentes, dans le catalogue de l'éminent éditeur anglo-saxon Routledge: en extrait dans son City Reader (Geddes 1996) et en fac-similé dans deux de ses collections documentaires et historiques - dans la collection «Social theory of the city ${ }^{1}$, du sociologue Bryan S. Turner (Geddes 1997); et dans la collection «Early urban planning: 1870-1940 $»^{2}$, de Richard LeGates et Frederic Stout (Geddes 1998).

Cependant, à y regarder de plus près, l'évidence de ce statut de classique n'est pas aussi limpide. «En avance sur son temps» pour de nombreux commentateurs, le livre a eu pourtant quelque mal à s'adapter aux différents contextes historiques de ses rééditions ultérieures. Jaqueline Mary Tyrwhitt (1905-1983), maître d'œuvre de l'édition de 1949, avançait elle-même l'idée que «le livre était presque déjà out of date au moment même de sa rédaction» (Tyrwhitt 1949 : ix). En introduction de son anthologie de 1972, Marshall Stalley (né en 1914) prévenait, quant à lui, le lecteur que «certains passages qui peuvent sembler, au public moyen et à l'administrateur public, ne plus avoir d'intérêt, ont été maintenus [...] pour leur importance historique» (Stalley 1972 : 109). Entre ces lignes, c'est la lisibilité même de Cities in Evolution qui est en jeu - mais les classiques sont-ils faits pour être lus? Stalley encourage pourtant le lecteur à tenter l'aventure, à faire cet effort en dépit du style de Geddes, "orné et discursif à l'excès » (ibid.: 109). Qualifiée de victorienne, de «carlyléenne» (Mumford 1944 : ix), voire de «sous-carlyléenne» (Grieve 1991 : 1943), sa prose a, en effet, toujours opposé certaines résistances aux lecteurs et aux traducteurs. Mais, au-delà de cette question stylistique, les éditeurs successifs du livre ont buté sur un autre type de difficulté: offre-t-il vraiment une synthèse de la pensée d'un Geddes tour à tour biologiste évolutionniste, sociologue-réformateur, philosophe-jardinier, géographe ou théologien? Suffit-il à rendre compte de celle-ci? Pierre Clavel (né en 1935), qui en a dirigé aux États-Unis une édition de poche, considé- 

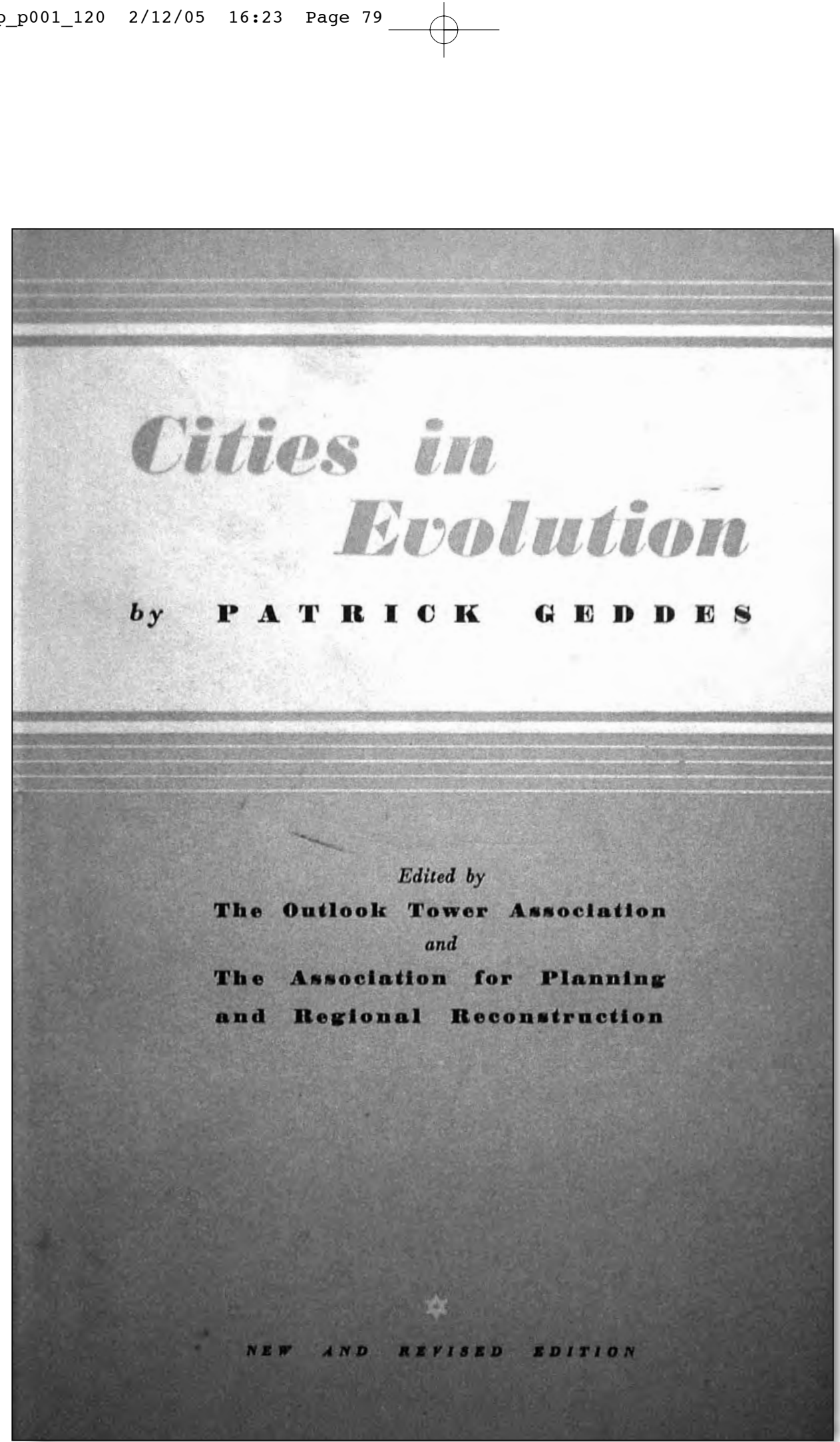

(ㄷ)

is

n

0

○

Couverture de la seconde édition de Cities in Evolution, édition abrégée dirigée par l'OTA et l'APRR (Londres, Williams \& Norgate, 1949) 
rait à juste titre que «le livre, comme la pointe d'un iceberg, s'appuie sur une masse énorme de substance, n'en élucidant qu'une partie» (Clavel 1971 : vii).

$\mathrm{La}$ fortune actuelle de cet ouvrage «incontournable» - qui resta pourtant près de trente-cinq ans sans être réédité - n'est donc pas sans soulever quelques questions. Parmi elles, se pose celle de sa plasticité, voire de son étrangeté disciplinaire. Comment expliquer l'aptitude de Cities in Evolution à s'inscrire dans des traditions aussi distinctes que l'urbanisme comme champ opératoire, la sociologie comme science ou même l'écologie comme doctrine? Cela tient-il au contenu même du livre, à la pensée de $\mathrm{P}$. Geddes ou bien à des malentendus plus ou moins volontaires, à des lectures fragmentaires? Le livre porte-t-il en lui tous ces fils entremêlés ou bien est-il inscrit de force dans ces généalogies? Une autre série de questions concerne la place et le statut de cet ouvrage particulier dans l'œuvre écrite de Geddes qui compte plus de deux cent cinquante titres (dont une quinzaine de livres). Pourquoi Cities in Evolution est-il le seul (ou presque ${ }^{3}$ ) à avoir atteint une telle postérité? Les raisons sont-elles, là encore, à trouver dans les qualités intrinsèques de l'ouvrage, garantes de sa survie face à une «sélection naturelle» éditoriale? Ces raisons, au contraire, sont-elles exogènes, liées à un choix conscient et délibéré par certains acteurs dans la bibliographie de Geddes?

Toutes ces questions portent en creux l'hypothèse centrale de cet article: le devenir de Cities in Evolution et de la «tradition" geddesienne qu'évoquait Gordon Cherry, doivent moins à l'action de $\mathrm{P}$. Geddes lui-même, qu'à celle, plus ou moins collective, de certains de ses «disciples» et/ou éditeurs posthumes: Arthur Geddes (1896-1968), Jaqueline M. Tyrwhitt, Percy Johnson-Marshall (19151993), Marshall Stalley, etc. Le rôle combiné de transmission, de diffusion, d'exégèse et parfois d'adaptation de l'œuvre de P. Geddes qu'ont joué ces divers acteurs est déterminant. Biographes et historiens l'ont, d'ailleurs, souvent évoqué, mais toujours pour le déplorer. Volker M. Welter, auteur du dernier livre en date sur l'œuvre du penseur écossais, le constatait lui-même: «[...] l'héritage de Geddes semble inviter à de régulières redécouvertes. Mais les tentatives pour le désigner comme un précurseur de problématiques ultérieures du vingtième siècle comme le régionalisme, l'environnementalisme ou la conservation du patrimoine architectural tendent non seulement à brouiller le contenu de ses idées mais à les retourner en leur exact opposé» (Welter 2002 : 251). Premier historien à rompre explicitement avec les approches «geddesiennes» de l'œuvre de P. Geddes, Volker $\mathrm{M}$. Welter considère l'action de ses exégètes successifs comme autant d'écrans qui nous voilent sa pensée authentique. Exactement à l'inverse, cette enquête s'intéressera, pour une fois, dans toute sa positivité à l'action déterminante de ces personnages abusivement qualifiés de secondaires et examinera historiquement, à travers eux, et à travers les successives rééditions ${ }^{4}$ posthumes du livre de 1915, comment s'est tissée cette «tradition» geddesienne, dont on a vu qu'elle n'allait pas de soi. 
J'ai acheté, pour ma part, L'Évolution des villes (Geddes 1994) au printemps 1994. Étudiant alors l'architecture et l'urbanisme, j'avais assisté quelques jours auparavant à un débat, qui accompagnait sa publication française. Les conditions, les modalités et le contexte de ce débat témoignent de la place de P. Geddes dans l'historiographie urbaine de manière suffisamment typique pour être rappelées ici. Le 7 mars 1994 à Beaubourg, dans le cadre hebdomadaire des «revues parlées de l'architecture et du design $»^{5}$, il réunissait trois architectes et historiens de l'architecture (Michael Darin, Marc Bédarida et Alexander Tsonis) et un géographe (Paul Claval). Rappelant les grandes lignes de la vie et de l'œuvre de P. Geddes, les interventions rendaient surtout, l'une après l'autre, un hommage à la «tradition geddesienne", à cette alternative urbanistique à laquelle son nom fut associé au cours du vingtième siècle: à la fois scientifique, évolutionniste, humaniste, communautaire, «eutopique», contextuelle, régionale, écologique, etc.

Au moment même de cette causerie, le centre Georges Pompidou était presque entièrement mobilisé par la grande manifestation sur «la Ville ${ }^{6}$ qui comprenait une grande exposition, des cycles de conférences et des programmes de films. Nulle part, pourtant, dans cette vaste rétrospective d'un siècle de culture urbaine européenne n'apparaissait l'auteur de la Cities and town planning Exbibition. À peine Anthony Sutcliffe, dans le catalogue, mentionnait-il son nom, précisant brièvement que "pour lui, l'urbanisme est fondé sur l'idée que tout plan d'urbanisme ne se conçoit pas sans les notions de croissance naturelle et de vie saine et libre de ses habitants» (Sutcliffe $1994: 128$ ). Seul finalement ce petit débat sur P. Geddes, confidentiel, externe à la manifestation générale, évoquait spécifiquement son œuvre, mais en la ramenant à sa composante urbanistique, et en s'appuyant sur la traduction française de Cities in Evolution, venue quatre-vingts ans plus tard.

Loin de résulter de l'effet du temps, cette présence-absence de P. Geddes de la scène officielle de l'urbanisme, cette reconnaissance marginale, ce rayonnement périphérique, me semble être une constante du personnage, déjà repérable de son vivant. En 1910, sa participation à la grande exposition londonienne du $\mathrm{RIBA}^{7}$ sur le town planning, préfigurait, en effet, ce triomphe latéral ou cet "échec magnifique» (Boardman 1978 : 429) de P. Geddes dans la sphère des architectes-urbanistes. Alors que ces derniers présentaient d'innombrables plans de villes au long des vastes galeries de la Burlington House, on confiait au «Professeur» une salle entière (mais minuscule et en impasse) pour présenter son civic survey d'Édimbourg: une collection hétérogène de documents graphiques, photographiques, cartographiques, destinée à rendre compte de la vie évolutive de cette ville dans ses aspects à la fois historiques, sociaux et géographiques; une accumulation inachevée, qualifiée de "cauchemardesque" par Patrick Abercrombie (1927 : 323), et qui marqua alors par sa singularité certains jeunes town- 
$\simeq \quad$ planners anglais. Si le survey geddesien offrait aux urbanistes du RIBA (Unwin, II Adshead, Lanchester, Lutyens, etc.) une méthode d'analyse à vocation scienti$\curvearrowleft \quad$ fique de l'organisme urbain, il ne fut jamais réellement adopté dans toutes ses a implications épistémologiques, c'est-à-dire comme instrument de la civics, cette (n) science civique que P. Geddes appelait de ses vœux depuis le début des années - $1900^{8}$ et dont il incitait à l'étude dans le sous-titre de Cities in Evolution.

\section{Un montage anachronique}

Avant d'évoquer les usages, il est nécessaire d'examiner un moment l'ouvrage qu'ils concernent, et que la tradition a figé dans l'apparence stable d'un classique. Une histoire, même superficielle, de ce livre montre pourtant à quel point son contenu est hétérogène, son écriture et sa publication ont posé problème. Tout commence par une commande de l'éditeur Williams \& Norgate pour sa nouvelle collection "Home university library of modern knowledge». Dirigée par Herbert Fisher, Gilbert Murray et John Arthur Thomson (proche collaborateur de Geddes) ${ }^{9}$, cette collection propose, à partir de 1911, des petits livres de vulgarisation scientifique, sur les thèmes les plus divers. Pour cette série destinée au grand public, Geddes et Thomson coécriront d'ailleurs trois volumes: Evolution ( $\left.\mathrm{n}^{\circ} 14,1911\right), \operatorname{Sex}\left(\mathrm{n}^{\circ} 85,1914\right)$ et Biology ( $\left.\mathrm{n}^{\circ} 111,1925\right)$. Cependant, le manuscrit que soumet P. Geddes en hiver 1912, malgré l'appui de son vieil ami, est catégoriquement rejeté par les directeurs de la collection (Meller 1990 : 191192). Ces derniers invoquent son contenu trop personnel, son inadéquation avec le format de la collection et surtout son inadaptation à un lectorat populaire ${ }^{10}$. Malgré tout, Williams, conscient de l'émergence de cette nouvelle préoccupation pour le town planning en même temps que du vide éditorial en la matière, propose immédiatement à Geddes de publier son ouvrage hors collection, dans une version élargie et illustrée. Il faut attendre 1915 pour que paraisse finalement Cities in Evolution, près de cinq années après les premiers pourparlers.

Une des principales difficultés de sa publication tient sans doute à ce que Cities in Evolution n'a jamais été écrit comme un livre. Le premier manuscrit est élaboré pendant l'année 1911 alors même que P. Geddes est presque entièrement absorbé dans la constitution, la promotion et les montages successifs de sa Cities and town planning Exbibition (à Chelsea, Édimbourg, Dublin et Belfast). Le temps qui lui reste est consacré à de nombreuses conférences et à leur publication dans les revues spécialisées (Sociological Review, town planning Review, Garden Cities E town planning Magazine, etc.). Trop peu d'espace est laissé à la plume - au demeurant laborieuse - de P. Geddes pour rédiger son opus syntheticum sur l'évolution des villes. Et ce que Geddes soumet en 1912 à la Home university library n'est autre qu'une compilation de ses multiples contributions au town-planning movement depuis le milieu des années 1900. 
Le second chapitre restitue la conférence de $\mathrm{P}$. Geddes au congrès de Birkenhead, organisé par Lord Leverhulme et le Royal Institute of Public Health, en juillet 1910 (Geddes 1911). Le chapitre iv reprend le contenu d'un article pour la town planning Review (Geddes 1912). Le chapitre ix est la synthèse de plusieurs comptes rendus de voyages d'étude en Allemagne, organisés notamment par le National Housing and Town Planning Council. Au cœur du chapitre xvi, nous retrouvons un document de travail élaboré par le cities committee de la Sociological Review, précisant la méthode du city survey. Fondé et dirigé par P. Geddes, ce comité constitue son principal ancrage institutionnel dans les premières années du town planning movement. C'est depuis cette base londonienne qu'il milite pour sa «science civique» et qu'il pilote certains surveys, en particulier celui de la ville de Chelsea (1908), dont la présentation publique est retranscrite dans le chapitre xvii de Cities in Evolution (Geddes 1908a). Les chapitres vii, xi, xii, xiii, xiv et xv, qui ne figuraient pas dans le manuscrit de 1912 complètent l'ouvrage avec différents textes témoignant de la principale préoccupation de Geddes dans cette première moitié des années 1910: les expositions d'urbanisme et les musées civiques. On retrouve ainsi une conférence donnée à l'université de Dundee en 1907 sur le «Musée et la ville»(Geddes 1908b), une conférence devant le cities committee sur l'exposition de Londres de 1910 ainsi que le guide-catalogue de l'«Exposition comparée des villes» de Geddes à Gand en été 1913 (Geddes 1913).

On le voit, ce livre est moins écrit que parlé. Qu'il s'agisse de ses conférences ou de ses longues visites guidées de la Cities Exhibition, c'est sa parole que P. Geddes a cherché à enregistrer: une suite d'exposés retranscrits, une suite de textes par lesquels le conférencier s'expose au lecteur. On pourrait dire que Cities in Evolution est plus une exposition qu'un livre, une autoexposition de son auteurorateur qui tente l'impossible traduction typographique de son verbe vivant. Chimère éditoriale, ce montage plus ou moins articulé vise finalement moins la théorisation d'une pensée que la pérennisation d'une présence. Dans ce recueil presque autobiographique, P. Geddes capitalise ses multiples activités, ses nombreux projets et ses quelques réalisations dans le champ naissant du town planning (Expositions, surveys, Outlook Towers, University Settlements, etc.) en même temps qu'il s'y met lui-même en scène. Bouclant un cycle, ce livre incarne physiquement Geddes au moment même où celui-ci s'absente de la scène européenne, au moment même où, au tournant de la soixantaine, il s'engage dans une vie lointaine de prophète-urbaniste en Inde. Tragiquement conscient de l'incompatibilité fondamentale entre sa vie et son livre, Geddes confie dans une «lettre d'orient» à sa femme, quelques mois avant sa parution, son «grand soulagement» d'avoir "complètement fini ce livre ennuyeux (Cities in Evolution)»" ${ }^{11}$.

Cette rapide analyse des raisons et des conditions d'apparition de l'ouvrage au tournant de la Première Guerre mondiale laisse apparaître plusieurs choses. D'abord ce livre, compilation provisoire et hétéroclite, n'en est pas vraiment un. D'autre part, c'est un objet étrange dans la bibliographie de Geddes composée 
essentiellement de textes courts, souvent tirés de conférences, ou bien de surveys, plus ou moins imposants, qui vont d'ailleurs dominer sa production dans ses années indiennes (1914-1924). Enfin, loin d'incarner un moment théorique fondateur pour le town planning, il prétend représenter la vie héroïque de son auteur au sein de cette sphère; une vie à la fois singulière et marginale, qu'il a voulu «exemplaire».

\section{Un culte hagiographique}

Lorsqu'en avril 1932, après avoir été tardivement anobli par le roi George V, Patrick Geddes cesse de vivre, son champ de rayonnement est certes vaste mais complexe et hétérogène. Dans l'épais supplément nécrologique de la Sociological Review, en octobre 1932, vingt-quatre contributeurs lui rendent hommage, dressant collectivement un portrait chinois du polygraphe écossais. Parmi eux, on compte seulement deux town-planners: Henry Vaughan Lanchester ${ }^{12}$ et George Lionel Pepler ${ }^{13}$, deux figures institutionnellement reconnues de l'urbanisme anglais. Si le second évoque l'importance de P. Geddes dans le développement du regional planning, le premier témoigne plutôt, dans sa contribution intitulée "town planning in India», d'une partie des activités indiennes de Geddes. Cependant, les deux urbanistes ne dérogent absolument pas au ton singulier, quasi biblique, que l'on adopte alors généralement pour parler de Patrick Geddes. Pepler évoque à plusieurs reprises, dans sa courte notice, sa mystérieuse influence sur les town-planners qui l'ont côtoyé: tout en estimant qu' «il est impossible d'expliquer clairement la nature de l'inspiration que l'on recevait de lui», il rappelle que "la trinité folk-work-place, qu'il défendait, représente aujourd'hui l'évangile de l'urbaniste, mais [que] nombreux en étaient ignorants avant qu'il ne leur ouvre les yeux» (Pepler 1932 : 382). Adoptant le même ton prophétique, Lanchester nous prévient: "Ceux qui lisent son Cities in Evolution sont informés de son approche de la question mais, même avec ce guide, il reste beaucoup à découvrir, que seul peut atteindre celui qui a eu la bonne fortune d'avoir collaboré avec lui à maintes occasions» (Lanchester 1932 : 370).

Qu'ils soient urbanistes, économistes, géographes, sociologues, éducateurs, réformateurs, etc., qu'ils soient célèbres ou anonymes, collaborateurs, élèves ou amis de Geddes, les divers auteurs de ce Sheaf of Tributes semblent tous mus par une même nécessité: témoigner de sa parole, de ses actions, pour transmettre ce qui est, au fond, l'essentiel de son œuvre, c'est-à-dire, sa vie elle-même. La nécessité biographique, quasiment apostolique, qui s'exprime là, est une constante de l'historiographie de P. Geddes, dès ses origines. Le premier ouvrage qui lui est consacré et qui paraît de son vivant porte, à cet égard, un titre éloquent: The Interpreter Geddes: the Man and his Gospel (Defries 1927).

S'il y a une "tradition geddesienne", elle se tisse d'abord au sein de cette famille, composée de ceux qui ont connu P. Geddes, de ceux qui, pour des rai- 
sons et à des degrés divers, ont engagé cette connaissance dans des stratégies personnelles ou collectives, de ceux qui ont capitalisé, dans ce culte hagiographique de Geddes, la parcelle de leur vie passée à ses côtés. Cette famille, au sein de laquelle se distinguent des individus et des groupes, s'identifie par une posture filiale au vieux "Professeur», même si celle-ci peut se formuler de multiples manières selon les cas. Ces phénomènes relativement courants se manifestent avec une ampleur particulière dans le cas de Geddes, qui, à défaut de fonder une nouvelle discipline, s'est toujours attaché de nouveaux disciples, misant sur eux pour sa propre postérité, «comme le coucou qui laisse ses œufs dans le nid des autres oiseaux, content que d'autres les nourrissent et s'en occupent jusqu'à ce qu'ils puissent voler de leurs propres ailes ${ }^{14}$.

\section{Un fils geddesien}

La réédition en 1949 de Cities in Evolution est un terrain particulièrement fécond pour observer la manière dont se distinguent et s'articulent les sous-groupes de cette nombreuse famille geddesienne. La page de garde nous indique que le livre est édité sous les auspices de l'Outlook Tower Association (Édimbourg) et l'Association for Planning and Regional Reconstruction (Londres) ${ }^{15}$ deux des principales sphères où l'on faisait usage de $\mathrm{P}$. Geddes dans les années 1940.

Empruntant son nom au musée-laboratoire-observatoire civique que P. Geddes installa dans les années 1890 dans une tour du vieil Édimbourg (Chabard 2001 : 64-89), l'OTA a été fondée en 1905 par un groupe d'amis et de proches collaborateurs. Au moment où les activités de Geddes se déplaçaient à Londres - autour de la Sociological Society - il s'agissait alors de sauver la tour de la faillite, de la réaménager et de l'exploiter en vue d'une gestion financière plus équilibrée. Fédérant l'énergie de quelques disciples et amis, l'association restera l'organe de gestion des entreprises édimbourgeoises de Geddes, en son absence. Après sa mort en 1932, l'OTA ne change pas fondamentalement de fonction en devenant le lieu privilégié de pérennisation et de patrimonialisation de son nom et de son œuvre. Au sein du très familial comité exécutif on compte de fidèles disciples comme Herbert John Fleure (1877-1969) ou Edward Mc Gegan, mais aussi sa propre fille, Norah (1887-1967) et Frank C. Mears (18801953), son gendre et principal collaborateur pour le civic survey d'Édimbourg et la Cities Exhibition (1911-1919), alors président de l'association. On trouve également son fils cadet Arthur (1895-1968) qui va s'affirmer comme un des principaux moteurs de diffusion de son œuvre dans les années 1935-1955, en dépit d'une problématique et insatisfaisante relation filiale ${ }^{16}$.

Fraîchement installé à Édimbourg, ayant obtenu un poste en géographie à l'université, il rejoint l'OTA au début des années 1930. La tâche est alors immense. D'une part, les réalisations geddesiennes (Outlook Torwer, Scots College, 
etc.) sont dans une condition matérielle et financière déplorable. D'autre part, la reconnaissance dont Sir Patrick Geddes jouissait dans les années 1910 chez les town-planners, les sociologues, les géographes ou même les biologistes, s'est quelque peu fanée, ces domaines ayant connu, entre-deux-guerres, d'importants renouvellements institutionnels, épistémologiques et générationnels. L'édifice geddesien reste donc, pour une grande part, à construire et c'est, délibérément, le programme que s'assigne l'OTA. Mais si, dès 1932, des actions concrètes sont entreprises en vue de pérenniser son œuvre, de rassembler et classer ses archives éparses, de rééditer ses livres et de publier ses nombreux manuscrits inédits ${ }^{17}$, ce n'est qu'après-guerre que, pour une part, elles trouveront leur aboutissement.

La libération de la France permet d'abord à Arthur Geddes, devenu président de l'OTA, de clore définitivement le cycle montpelliérain de la vie de son père ${ }^{18}$ et d'exploiter les nombreux matériaux bloqués là par la guerre: ses papiers, sa seconde Cities and town planning Exbibition. Outre de nombreux travaux entrepris dans la tour à partir de 1947, l'agenda de l'association, orchestré par Arthur, comporte une longue liste de publications geddesiennes. En décembre $1947^{19}$, cette liste accompagne une lettre circulaire qu'Arthur Geddes adresse à la presse et à d'éventuels éditeurs ${ }^{20}$. Pas moins de dix projets d'ouvrage la composent, exploitant les différents éléments de l'héritage paternel: ses surveys, ses multiples cours et conférences, son innombrable correspondance, sa surabondante production graphique et diagrammatique, sans compter ses nombreux manuscrits inédits.

De cet optimiste programme éditorial, exploitant les multiples facettes de l'œuvre de P. Geddes (sociologie, biologie, géographie, philosophie, réformisme social, urbanisme, etc.), ne résultera finalement que trois ouvrages. Le dernier, intitulé Pionneer of Sociology (Mairet 1957), qui clôt en 1957 cette décennie geddesienne, est une biographie épistolaire, qui figurait dans la liste de 1947. Éditée et rédigée par Philip Mairet ${ }^{21}$, elle entend compléter l'ouvrage de Philip Board$\operatorname{man}^{22}$ (Boardman 1944), seul de la période à avoir échappé au contrôle d'Arthur Geddes ${ }^{23}$.

\section{Une convergence d'usages}

Les deux autres ouvrages publiés alors concernent exclusivement le Geddes town-planner. Il s'agit, en 1947, du petit recueil d'extraits de ses surveys indiens (Tyrwhitt 1947) et, en 1949, de la réédition de Cities in Evolution, après trentequatre ans. Cette sélection dans la liste de 1947 (et dans la bibliographie de P. Geddes) ne procède ni du hasard, ni d'un choix délibéré d'Arthur Geddes, mais répond au programme d'une autre sphère de la famille geddesienne: le town-planning anglais et plus précisément un groupe d'urbanistes gravitant autour de l'APRR et envisageant la figure de Geddes comme une référence opératoire dans leurs débats de l'après-guerre. C'est la rencontre de ces deux 
branches, l'une familiale, l'autre professionnelle, c'est la convergence ponctuelle et provisoire de leurs intérêts qui décidera de ces deux publications, dont on peut penser qu'elles ne figuraient pas parmi les priorités d'Arthur Geddes - plus enclin à exploiter ses propres travaux d'édition des conférences de son père traitant de la civilisation contemporaine ou de la signification sociale de la mythologie grecque.

Dans une lettre à Lewis Mumford, datée du 6 octobre 1944, Frederic J. Osborn (1885-1978) raconte sa rencontre, en Écosse, avec les membres de l'OTA : «J'ai à nouveau soulevé la question de la réédition de Cities in Evolution mais ils avaient tous l'air très indécis à ce sujet. Un travail d'édition est certainement nécessaire ainsi qu'un nouvel appareil d'illustration. Mais si le travail est bien fait et si quelqu'un écrit une nouvelle introduction, je pense qu'il y aurait un public pour ce livre en Angleterre et aux États-Unis» (Hughes 1972: 63). C'est finalement J. M. Tyrwhitt qui réalisera, en 1949, ce projet latent. Active au côté d'Osborn au sein de la TCPA dans la deuxième moitié des années 1930, membre du groupe MARS à partir de 1941, cette paysagiste-urbaniste d'origine sud-africaine est surtout un des membres fondateurs et l'animatrice principale de l'APRR. N'ayant jamais physiquement rencontré P. Geddes, son intérêt pour sa pensée et ses écrits n'est ni historique ni patrimonial mais résolument instrumental et professionnel. À l'heure de la seconde reconstruction, il s'inscrit dans l'agenda opérationnel de l'APRR, pensée comme un relais entre les prescriptions des nouvelles législations urbanistiques et la pratique effective d'un nouveau corps de planners. Siège, dès 1940, de la School of Planning and Research for Regional Development, dissidente de l'Architectural Association et habilitée par le RTPI à délivrer un diplôme d'urbaniste, l'association élabore et publie manuels, atlas, surveys, brochures d'information, ouvrages pratiques et utilitaires, et multiplie, principalement dans la deuxième moitié des années 1940, les colloques, les expositions à l'usage des praticiens. Professionnelle sans être technocratique, il règne, dans cette association dominée par des personnalités telles qu'Eric A. Rowse et Jaqueline M. Tyrwhitt, une vision sociale et humaniste de l'urbanisme, un idéal civique très en phase avec l'humeur anglaise de l'immédiat après-guerre.

La réédition, par l'APRR, des écrits de $\mathrm{P}$. Geddes répond directement à cette nécessité de reformulation de la profession d'urbaniste, à la fin des années 1940: en proposant, d'abord, un modèle méthodologique alternatif, déjà constitué, puis en légitiment historiquement les prescriptions de l'APRR, par le biais d'une figure tutélaire. À cet égard, la publication par J. M. Tyrwhitt de Patrick Geddes in India (Tyrwhitt 1947) est exemplaire. Dans ce petit "Geddes de poche» (Boardman 1948 : 10) d'une centaine de pages, elle propose une anthologie de courts fragments de ses imposants surveys indiens. Abstraits de leur contexte d'origine, ces textes sont organisés selon sept grands chapitres thématiques, derrière lesquels se lisent clairement les préoccupations de l'APRR: «The Diagnostic Survey», "Conservative Surgery», "A Sociological Approach», «Open Space and Trees», etc. 
On décèle la même volonté instrumentale dans l'édition «nouvelle et révisée» de Cities in Evolution, en 1949. "Alors que la loi de $1947^{24}$ est pleinement entrée en application et que de vastes plans de développement sont en préparation partout dans les Îles britanniques, ce livre - source des courants les plus féconds de la pratique urbanistique - fait une opportune réapparition» annonce la brochure promotionnelle. Moins qu'un document historique, précautionneusement exhumé du passé, c'est un véritable guide pour le présent que l'APRR entend proposer à ses contemporains. Et cette actualisation du livre, avec un titre simplifié, se fait au prix d'un important travail éditorial, d'une suite d'ajouts et de retraits, qui le rendent «à la fois plus grand et plus court que le texte original» (Mumford 1950 : 82). Celui-ci subit de multiples corrections, mises à jour, enrichissements typographiques, etc.; certaines informations sont insérées (dates biographiques, historiques); des nuances lexicales sont apportées (un must devient could, un primarily devient largely, etc.); les titres de chapitres sont raccourcis; les petits synopsis qui les suivaient sont fragmentés et redistribués dans le fil du texte, sous la forme d'intertitres; le rythme de la lecture est également modifié par un nouveau découpage des paragraphes.

Outre ces multiples aménagements et adjonctions, Jaqueline M. Tyrwhitt a opéré, sous le contrôle d'Arthur Geddes et de George L. Pepler, à des coupes franches, à des amputations, parfois de quelques lignes, parfois de plusieurs pages, voire de chapitres entiers: sur les quatre cent six pages d'origine, pas moins de cent soixante sont supprimées. L'analyse détaillée du contenu de ces coupes en fait apparaître trois grands types. D'abord Tyrwhitt a allégé le texte de certains passages où Geddes s'aventure trop loin du town-planning: un détour sur la littérature utopienne (Geddes 1949 : 87-88), une critique politico-économique du capitalisme (ibid.: 68-69), une digression sur l'usage du survey dans les écoles primaires (ibid.: 335-336), etc. Elle a ensuite censuré systématiquement les pages intolérables pour un lecteur anglo-saxon d'après-guerre: par exemple, les deux chapitres sur l'urbanisme allemand (chap. IX et X). Enfin, la plupart des coupes concernent les passages trop datés, où $\mathrm{P}$. Geddes parle au présent des évènements et des acteurs du townplanning movement des années 1900-1910: projets, expositions, voyages d'étude, congrès, législations, etc. Les trois chapitres sur l'habitat ouvrier, obsolètes à l'heure de la seconde reconstruction, sont ainsi totalement supprimés. Pour des raisons semblables, J. M. Tyrwhitt déshabille le texte de l'intégralité de ses illustrations originales. Pour actualiser ce livre, pour le rendre lisible dans un autre temps, c'est comme s'il fallait l'arracher au sien. Dépouillée de ses ancrages temporels, il ne reste de la pensée de Geddes que son idéalisme anachronique, celui qui l'isola souvent de ses contemporains, celui qui la rend, peut-être, disponible à tout usage ultérieur.

La réussite de cette édition de Cities in Evolution - de toutes sans doute la plus lue - tient à cette simplification du noueux texte original mais également à 
l'adjonction d'un important appareil d'annexes: nouvelle introduction de J. M. Tyrwhitt, qui vient doubler la préface de Geddes; nouvel index thématique; notice biographique; analyses explicatives des thinking machines de Geddes ${ }^{25}$, à l'heure où la «Grille C.I.A.M.» est en débat. Mais l'annexe la plus détaillée concerne la Cities and town planning Exbibition, dont la vente éventuelle à l'APRR inaugure, en 1946, la collaboration de Jaqueline M. Tyrwhitt et George L. Pepler avec Arthur Geddes. L'exposition, rapatriée de Montpellier en octobre 1947, est stockée au ministère du Town and Country Planning à Londres. La transaction n'aboutit pas mais les deux urbanistes de l'APRR sélectionnent dans ce vaste corpus une quarantaine de documents pour les exposer en novembre 1948 à l'International Housing and town planning Exhibition (Olympia, Londres). Le catalogue de cette exposition réduite constitue, dans Cities in Evolution, une annexe illustrée de trente et une pages, émaillée d'extraits du texte-guide de Geddes de 1911 et d'un développement de G. L. Pepler sur les cités-jardins, en tant qu'ancêtres des new towns.

\section{Un père de l'urbanisme}

La publication de Cities in Evolution, en 1949, constitue un point crucial dans l'historiographie de Patrick Geddes car elle marque une inflexion décisive dans les usages posthumes de celui-ci. Au cours de la décennie 1945-1955 s'essoufflent lentement, par un effet générationnel, les entreprises de ses plus énergiques descendants hagiographes; ceux qui tentent d'entretenir les braises déclinantes de l'héritage paternel, de prolonger artificiellement l'écho de sa voix prophétique. Au contraire, prenant en quelque sorte l'ascendant sur les descendants, cette décennie voit le redéploiement d'un autre type de geddesiens: des architectes-urbanistes, qui, sans l'avoir nécessairement connu personnellement, et à un moment critique de leur définition professionnelle, font un usage instrumental de l'œuvre urbaniste du penseur écossais. C'est un tout autre Geddes qui se trouve alors remodelé. Tandis que les premiers insistaient sur l'absolue et inclassable singularité du personnage, encyclopédiste touche-à-tout, rétif à toute assignation disciplinaire et institutionnelle, les seconds sélectionnent au contraire dans son œuvre ce qui est appropriable, ce qui répond aux problématiques spécifiques de leur champ: l'articulation entre savoir et action par le survey, la définition épistémologique de l'urbanisme, les échelles et les limites de la planification, etc. Échappant à un probable déclin, c'est au sein de l'urbanisme que la «tradition geddesienne» va ainsi se recentrer et se prolonger au prix d'une adaptation disciplinaire.

Lors de la célébration du centenaire de Patrick Geddes, en octobre 1954 moment culminant de l'activité de l'OTA - se confirme cette équation. Organisateur de l'évènement, Arthur Geddes programme une journée de colloque où il convie quatre intervenants: le généticien Francis A. E. Crew (1886-1873) et le 
géographe, membre de l'OTA, Herbert J. Fleure, deux témoins, déjà âgés, de la II vie du vieux «Professeur»; les urbanistes Patrick Abercrombie et William Hol— ford, deux membres éminents du $\mathrm{RTPI}^{26}$. Ce portrait à quatre voix (Sir Patrick Geddes Centenary... 1954) traduit ce déplacement progressif entre un Geddes geddesien et un Geddes planner, entre patrimonialisation et actualisation de son œuvre, entre les arrangements d'une approche biographico-patrimoniale et les distorsions d'un usage «mono-disciplinaire».

Ce déplacement se lit clairement dans les rééditions ultérieures de Cities in Evolution, et en particulier dans les trois suivantes qui, entre 1968 et 1972, jalonnent une nouvelle phase de redécouverte et d'usage de l'œuvre de Patrick Geddes. Publiée en 1968, la première est due à l'architecte-urbaniste Percy Johnson-Marshall (1915-1993). Étudiant de Reilly et d'Abercrombie à Liverpool, dans les années 1930, abonné au cours par correspondance de la SPRRD de J. M. Tyrwhitt pendant sa mobilisation, P. Johnson-Marshall représente la figure typique du planner anglais d'après-guerre, à la fois expert affûté, tenant d'un savoir technique sur la planification, et humaniste convaincu, révérant la figure d'un Geddes largement reconstruite par la génération précédente ${ }^{27}$. Invité en 1959 par Robert Matthew (1906-1975) pour enseigner dans son tout nouveau département d'architecture à l'université d'Édimbourg, il y devient professeur d'urbanisme en 1964. Sa réédition de l'ouvrage de $\mathrm{P}$. Geddes prend son sens dans ce contexte universitaire, et notamment dans le cadre de la School of the Built Environment fondée par R. Matthew en 1967, rassemblant les départements de Landscape Architecture, de géographie et celui d'Urban Design and Regional Planning dirigé par P. JohnsonMarshall. Ce dernier choisit de publier respectueusement Cities in Evolution en fac-similé, armé simplement d'une nouvelle introduction, qui se présente essentiellement comme une lecture du contenu de chacun des chapitres, de ce qu'il convient d'y trouver et de ce qui y reste opératoire pour le présent.

Le fac-similé est également la formule éditoriale choisie par Pierre Clavel, en 1971, pour la réédition (au format poche) du livre culte de P. Geddes. Il est intéressant, à cet égard, de comparer son introduction avec celle de P. Johnson-Marshall, écrite au même moment à propos du même ouvrage. Alors que l'Écossais insiste sur la pertinence des prophéties urbaines de Geddes, sur sa prescience des conurbations, des mégalopoles et de l'urban sprawl, sur la légitimité de sa vision environnementale et régionale, c'est un autre Geddes que déploie l'Américain. Économiste de formation, auteur d'une maîtrise de regional planning sur Geddes (Clavel 1959) à l'Université de Caroline du Nord ${ }^{28}$, P. Clavel enseigne, dès 1967, à l'université Cornell, titulaire d'un doctorat sur l'implication des communautés dans les processus de planification (Clavel 1966). En 1971, il présente essentiellement Geddes sous un angle sociopolitique, comme un sociologue impliqué dans la Cité et comme un précurseur des théories de la «participation». A travers la figure de Geddes, il légitime l'engagement citoyen des sciences sociales, qu'il entend lui-même incarner. Selon lui, Cities in Evolution «suggère de telles théo- 


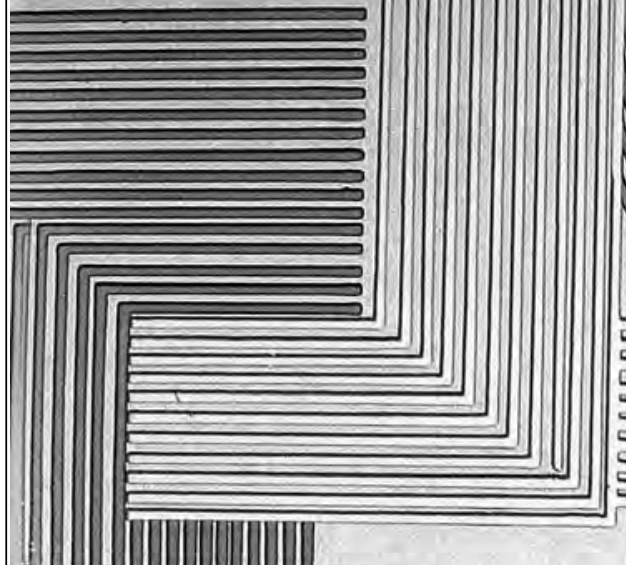

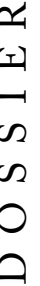

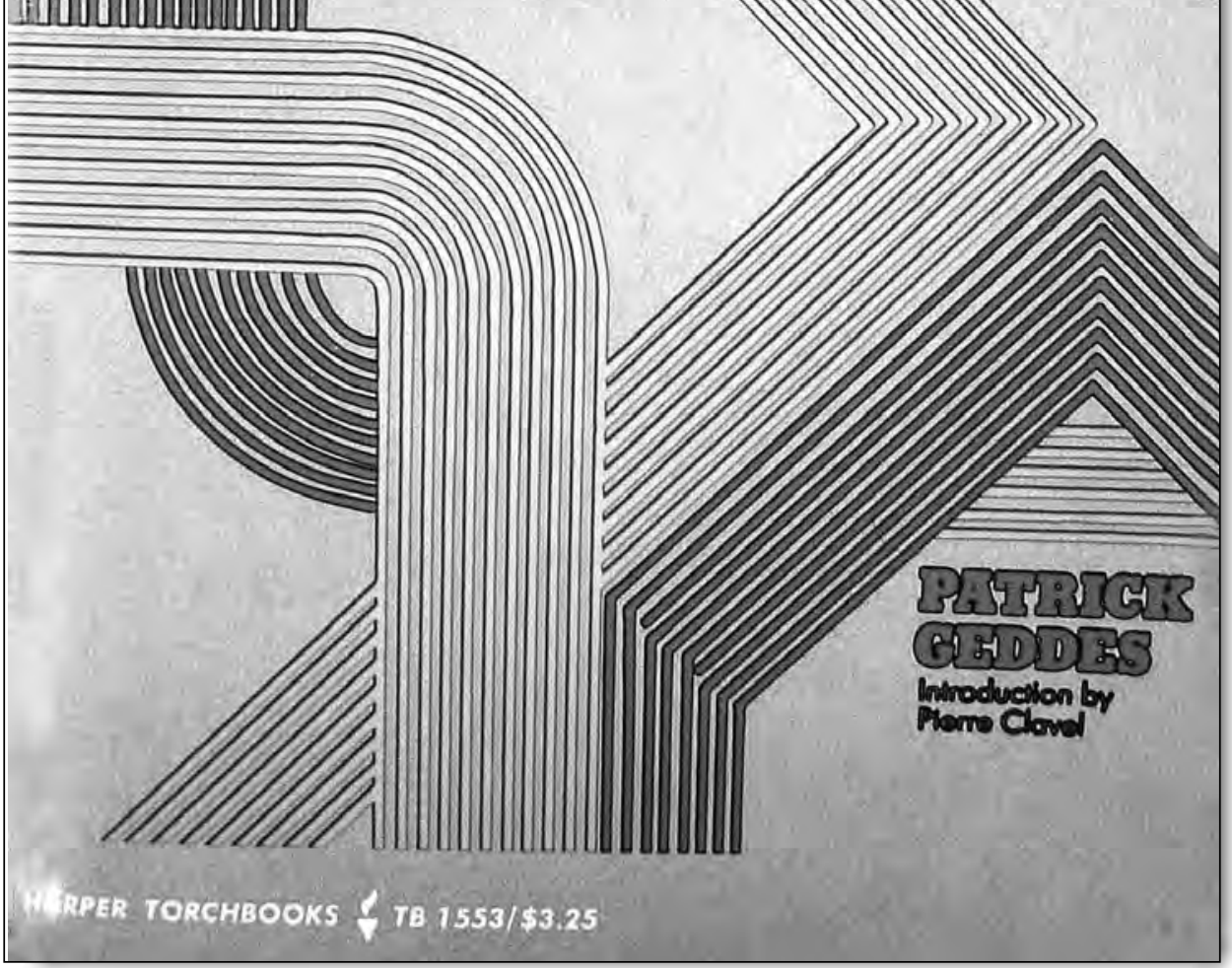

Couverture de l'édition de poche de Cities in Evolution, dirigée par Pierre Clavel (Londres, Harper Torchbooks- New York, Dover, 1971) 
ries et fonde tant leur possibilité que leur désirabilité. Si les théories ne sont pas abouties, et si les exemples invoqués sont un peu désuets, le livre offre un commencement et nous encourage à assumer la tâche» (Clavel 1971 : xxiii).

L'année suivante, Cities in Evolution connaît une nouvelle réédition, aux presses de l'université Rutgers. L'ouvrage de P. Geddes s'inscrit alors dans une anthologie rassemblant également ses six Talks from my Outlook Tower ${ }^{29}$ et de larges extraits de son rapport sur la ville de Lahore (Geddes 1917). L'enjeu, pour M. Stalley, maître d'œuvre de ce Patrick Geddes: Spokesman for Man and Environment, est de rendre disponible au lecteur américain les écrits de celui qu'il considère comme un prophète de l'environnementalisme: "Aux étudiants et praticiens du planning et au corps grandissant des écologistes, Geddes a laissé une philosophie éloquente, une méthodologie valide et un héritage pratique» (Stalley 1972 : xiv). Actif pendant quinze ans dans la planification des ressources et des paysages naturels de la région de Pittsburgh, engagé à partir de 1961 dans une carrière universitaire au Urban Studies Center de l'université Rutgers, ce sociologue de formation participe, au moment de cette réédition, à la fondation du Cook College, qui centralisera, dès 1973, les enseignements de science, d'agronomie et d'environmental studies de l'université. Très instrumentale, la démarche de M. Stalley ressemble à celle de J. M. Tyrwhitt, en ce qu'elle aboutit à une version abrégée du texte. Pour décrire son travail d'édition il emploie la métaphore geddesienne de «chirurgie conservatrice», nécessaire ablation des parties obsolètes d'un organisme (urbain ou éditorial) pour qu'il reste en vie (et lisible pour le lecteur cible: un étudiant américain en sciences de l'environnement).

$\mathrm{Au}$-delà des différences notables, ces trois rééditions convergent sur un certain nombre de points, et ces trois Geddes - le regional planner, le théoricien de la participation et le père de l'environnementalisme - participent d'un même personnage dont la construction est à l'œuvre depuis les années 1940. La principale similitude entre ces trois Cities in Evolution tient à leur contexte: les milieux académiques, à des moments critiques de questionnement du champ du planning au sein du spectre changeant des disciplines. Dans les trois cas, les éditeurs évoluent dans des cadres universitaires récents, en cours de définition ou d'institutionnalisation, à la croisée fragile de plusieurs domaines de savoir et d'action. Dans les trois cas également, ils sont eux-mêmes engagés, comme praticiens, dans des processus effectifs de planification. Le détour par Cities in Evolution et par la figure éternellement marginale et héroïque de Patrick Geddes vient servir ainsi des parcours semblables, consolider des postures institutionnelles similaires. Cet usage disciplinaire, qui s'imposait déjà dans les années 1940, confirme sa prééminence au tournant des années 1970, privilégiant du polygraphe écossais le profil du planner, infiniment déclinable, capable de dissoudre, d'absorber tous les autres; et tissant, au fil des générations, une «tradition geddesienne», à la fois cause et effet de tous ces usages posthumes. 


\section{Repères biographiques}

1854

1874-1878 20-24 ans Études de biologie auprès de Thomas Huxley à la Royal School of Mines (Londres).

1878-1879 24-25 ans

1880-1887

1884-1896 30-42 ans

1888-1919 34-65 ans Titulaire de la chaire de botanique à l'University College, Dundee (université de Saint Andrews, Écosse); poste sur mesure où il n'enseignait qu'un trimestre, de mi-avril à mijuin, mais payé à mi-temps.

$1900 \quad 46$ ans Participe à l'exposition universelle de Paris. Organise l'École internationale de l'exposition (avec I'International Association for the Advencement of Science, Art and Education).

190349 ans Cofonde la Sociological Society de Londres, avec notamment Victor V. Branford (1864$1930)$; il y prononce ses trois conférences sur la Science Civique - «Civics as applied sociology |» (1904); "Civics as applied and concrete sociology II» (1905); «A suggested plan for a civic museum» (1906).

1903-1910 49-56 ans Participe aux débats du Town Planning Movement.

Publication de City Development (1904), décrivant sa proposition urbanistique pour la ville de Dunfermline (Écosse) pour le compte du Andrew Carnegie Dunfermline Trust.

191056 ans Contribue, avec son civic survey d'Édimbourg, à la Town Planning Exhibition du RIBA à Londres.

1911-1913 57-59 ans Nommé responsable de la Cities \& Town Planning Exhibition (C\&TPI Exh), exposition itinérante destinée à promouvoir le Town Planning et présentée à Chelsea, Édimbourg, Dublin, Belfast et Gand.

191460 ans $\quad$ Départ pour l'Inde. Perte de la C\&TPI Exh dans un naufrage.

1914-1923 60-69 ans Élabore de nombreux rapports sur des villes indiennes. Présentation d'une seconde version de la C\&TPI Exh dans plusieurs villes indiennes (Madras, Calcutta, Nagpur, Lucknow, Bombay) puis à Paris (exposition de la Cité reconstituée, été 1916). Décès d'Anna et d'Alasdair en 1917.

1920-1923 66-69 ans Crée et occupe la chaire de sociologie et de science civique à l'université de Bombay . La C\&TPI Exh y est installée de manière permanente.

192369 ans Donne ses «6 talks from my Outlook Tower» à New York, à l'invitation de la Regional Planning Association of America et de Lewis Mumford.

1924-1932 70-78 ans Installé à Montpellier, il y construit son collège des Écossais, institut para-universitaire à vocation internationale.

Plusieurs séjours en Palestine $(1919,1920,1925)$ pour des travaux urbanistiques (Jérusalem, Jaffa).

$1928 \quad 73$ ans $\quad$ Second mariage, avec Lilian Brown (187?-1936).

$1932 \quad 78$ ans anobli pour son œuvre éducatrice; meurt à Montpellier. 
II APRR: Association for Planning and Regional Reconstruction (Londres)

MARS: Modern Architecture Research Group (Londres)

NLS: National Library of Scotland (Édimbourg)

OTA: Outlook Tower Association (Édimbourg)
RIBA: Royal Institute of British Architects (Londres)

RTPI: Royal Town Planning Institute (Londres) TCPA: Town and Country Planning Association (Londres)

US: University of Strathclyde (Glasgow)

\section{Notes}

1. Rassemblant les écrits de précurseurs de la sociologie urbaine: William P. Capes, Frederic W. Maitland, Seebohm Rowntree, Roderick D. MacKenzie, Lewis Mumford.

2. Rassemblant les premières contributions théoriques au town planning: Ebenezer Howard, Charles M. Robinson, Nelson P. Lewis, John Nolen, Clarence Perry, Patrick Abercrombie et Thomas Adams.

3. Parmi les rééditions posthumes des écrits de Geddes on trouve aussi ses trois conférences à la Sociological Society sur la discipline qu'il appelait «civics» (1904-1905), ses six articles du Survey Graphics, intitulées "Talks from my Outlook Tower» (1925) ainsi que certains de ses «surveys» indiens (en particulier celui de Lahore, 1917).

4. Nous avons limité cette enquête aux rééditions en anglais.

5. Le programme annonçait d'ailleurs par erreur «Bel Geddes, architecte écossais».

6. «La Ville: art et architecture en Europe, 18701993", 10 février-9 mai 1994 (commissaires: Jean Dethier et Alain Giheux).

7. On trouvera une liste des sigles en fin d'article.

8. Voir la série de trois conférences sur la civics qu'il prononce devant la Sociological Society, en 1904-1905.

9. Sir John Arthur Thomson (1861-1933), biologiste écossais, titulaire (1900-1930) de la chaire d'histoire naturelle de l'université d'Aberdeen. Il est coauteur des principales publications scientifiques de Patrick Geddes: The Evolution of Sex (1889), Evolution (1911), Problem of Sex (1912), Sex (1914), Biology (1925), Life: Outlines of General Biology (1931).

10. Voir Lettre de G. H. Perris, assistant d'édition, à Geddes, 18 mars 1912 (Kitchen 1975 : 240).
11. Lettre de Patrick Geddes à Anna Morton, 4 octobre 1914 (NLS, Ms. n 10504.78-90), citée par Giovanni Ferraro (1998 : 16).

12. Reconnu à la fois comme architecte et comme urbaniste, Henry V. Lanchester (1863-1953) est, en 1913, un des membres fondateurs du RTPI. Enseignant l'urbanisme à Londres et à Liverpool dès 1910, il est l'auteur de town planning in Madras (1918), Talks on town planning (1924) et The Art of town planning (1925).

13. Ingénieur de formation, George L. Pepler (1882-1959) compte également parmi les fondateurs du RTPI, dont il fut président en 19191920. En 1914 il entre au Local Government Board, et s'occupe des Greater London Arterial Road Conferences. En 1919, il devient Chief town planning Inspector au ministère de la Santé.

14. Geddes cité par Lewis Mumford (1944 : viii).

15. Fondée en 1939 à l'instigation de l'architecte Eric Anthony Ambrose Rowse (1896-c1982), l'APRR est une association à but non lucratif rassemblant des experts autour des problématiques urbaines et sociales de la Reconstruction.

16. En 1968, au terme de sa vie, Arthur Geddes avouera lui-même à Philip Boardman: "Je me souviens combien j'ai été triste en ouvrant le télégramme annonçant sa mort, combien j’ai été triste de ne ressentir qu'un immense soulagement " (Boardman 1978 : 445).

17. Pour financer ces actions, l'association lance, en 1935, un large appel de fonds, sous le label «Memorial to the late Profesor Sir Patrick Geddes » et sous l'égide de personnalités influentes: Patrick Abercrombie, Raymond Unwin, Henri Bergson, Georges Bernard Shaw, Rabindranath Tagore, D'arcy W. Thompson, etc.

18. Le «Plan des Quatre-Seigneurs», domaine comportant le collège des Écossais, avait déjà été 
vendu en juin 1943 au Commissariat régional des chantiers de la jeunesse du gouvernement de Vichy. 19. Avec notamment le soutien financier de la Fondation Carnegie.

20. Outlook Tower Association, «Proposed Books on Sir Patrick Geddes' Work» (Ts.), Édimbourg, s. d. [décembre 1947] (US, TGED 20-1-85).

21. Philip(pe) Auguste Mairet (1886-1975) est un intellectuel londonien proche du mouvement Arts \& Crafts, des milieux fabiens et socialistes chrétiens. En 1908, il rencontre Geddes qui milite alors pour la reconstruction du Crosby Hall à Chelsea. En 1924, il collabore, comme dessinateur, à son exposition «Hall of Vision» à la School for Oriental Studies. Ami et biographe d'Alfred R. Orage (1875-1934), il lui succède, à sa mort, à la rédaction en chef de The New English Review.

22. En 1926, Philip Boardman (1906-1989), jeune étudiant américain, rencontre Geddes à Montpellier. Il passe l'année scolaire 1929-1930 au collège des Écossais où il soutient, en 1936, une thèse sur l'œuvre éducatrice du penseur écossais.

23. Ce livre est dû à l'action moins ostensible d'un autre «fils geddesien» : Lewis Mumford, qui soutient et préface l'ouvrage. Ayant toujours gardé ses distances face aux désirs de Geddes de faire de lui son «fils spirituel», Mumford ne se départira jamais d'un rapport ambigu et contradictoire envers ce maître stimulant mais envahissant, ce père généreux mais tyrannique, ce penseur ambitieux mais confus. Personnage attentif et influent des milieux geddesiens, il se tiendra toujours en retrait de leurs nombreuses entreprises éditoriales, se contentant du rôle clé d'expert-préfacier. Au croisement du contexte anglais et américain, des milieux de l'architecture, de l'urbanisme et des sciences humaines, L. Mumford traversera également par sa longévité les différentes périodes de redécouverte et d'usage de Geddes (Novak 1995).

24. Le Town and Country Planning Act, adopté en août 1947, en application le $1^{\text {er }}$ juillet 1948.

25. Notamment un texte préparée par John F. C. Turner (né en 1927), futur urbaniste alors jeune étudiant en architecture à l'Architectural Association, assisté de Paffard Keating-Clay, étudiant en mathématiques.

26. William Holford (1907-1975) est alors le président du RTPI. Récipiendaire de la Médaille d'or de cet Institut en 1955, Patrick Abercrombie (1879-1957) en fut le président en 1925-1926.

27. En 1968, en même temps que cette réédition, Percy Johnson Marshall est l'initiateur d'un projet qui ne se réalisera que vingt ans plus tard: la mise en place dans l'Outlook Tower d'un « Patrick Geddes Centre for Planning Studies».

28. Là où Philip Boardman publia sa biographie de Geddes en 1944.

29. Publiés dans The Survey Graphic entre février et septembre 1925, ces six textes sont issus des conférences que Patrick Geddes, à l'invitation de Lewis Mumford, a donné à la New School for Social Research de New York, en été 1923.

\section{Références}

u Abercrombie, L. Patrick. 1927. «Geddes as Town-Planner", in Amelia Defries, The Intepreter Geddes: the Man and his Gospel. Londres, Routledge: 322-325.

u BoARdman, Philip. 1944. Patrick Geddes: Maker of the Future. Chapel Hill, University of North Carolina Press.

- 1948. «Not Housing but Home-building: the life-centered approach of Patrick Geddes», Teknisk ukebald (Oslo), n 33: 1-15.

- 1978. The Worlds of Patrick Geddes: Biologist, Town planner, Re-educator, Peacewarrior. Londres, Routledge \& Kegan.

u CHABARD, Pierre. 2001. «L'Outlook

Tower, anamorphose du monde: Patrick
Geddes et le thème de la vision", Le Visiteur, $\mathrm{n}^{\circ}$ 7: 64-89.

u Cherry, Gordon E. 1974. The Evolution of British Town Planning. Londres, Leonard Hill Books.

u Clavel, Pierre. 1959. «The contribution of Patrick Geddes to the theory of city and regional planning», Th.: City and Regional Planning, University of North Carolina, Chapel Hill.

- 1966. «The genesis of the planning process: experts and citizen boards in Sullivan County, New York», PhD, université de Cornell, Ithaca.

- 1971. «Introduction to the Torchbook 
Edition », in Patrick Geddes, Cities in Evolution. Londres, Harper TorchbooksNew York, Dover: vii-xxiii.

u DeFries, Amelia Dorothy. 1927. The Interpreter Geddes: the Man and his Gospel. Londres, Routledge.

u Ferraro, Giovanni. 1998. Reiducazione alla speranza: Patrick Geddes in India, 19141924. Milan, Jaca Book.

u GedDEs, Patrick. 1908a. «Chelsea, Past and Possible», Sociological Review, vol. $1, \mathrm{n}^{\circ}$ 4: 357-363.

- 1908b. «The Museum and the City: a practical proposal», The Museum Journal, mai: 371-382.

- 1911. "City Survey for Town Planning», Journal of the Royal Insititute of Public Health, février: 79-90.

- 1912. "The two-fold aspect of Industrial Age», Town Planning Review, vol. 3, n 3: 176-187.

- 1913. Two Steps in Civics: "Cities and Town Planning Exhibition» and the "International Congress of Cities", Ghent International Exhibition, 1913. Liverpool, The University Press.

- 1915. Cities in Evolution: an introduction to the Town Planning Movement and to the Study of Civics. Londres, Williams \& Norgate.

- 1917. Town Planning in Labore: A report to the Municipal Council. Lahore, Commercial Printing Works.

- 1934 [1915]. Ciudades en Evolucion, par E. L. Revol. Buenos Aires, Thornton Butterworth (éd. orig. Cities in Evolution. Londres, Williams \& Norgate).

- 1949 [1915]. Cities in Evolution (édition nouvelle et révisée par l'Outlook Tower Association, Édimbourg, et l'Association for Planning and Regional Reconstruction, Londres). Londres, Williams \& Norgate-New York, Oxford University Press.

- 1960 [1915]. Ciudades en Evolucion, par E. L. Revol. Buenos Aires, Ed. Infinito (éd. orig. Cities in Evolution. Londres, Williams $\&$ Norgate).

- 1965 [1915]. «L'Évolution créatrice des villes» (extraits de Civics as applied Sociology et de Cities in Evolution), in Françoise Choay (éd.), L'Urbanisme, utopies et réalités : une anthologie. Paris, Seuil: 345-53.

- 1968 [1915]. Cities in Evolution, an Introduction to the Town Planning Movement and to the Study of Civics, (introd. Percy JohnsonMarshall). Londres, Ernest Benn-New York, Howard Fertig.

- 1970 [1915]. Città in Evoluzione (préf.

Carlo Carozzi), par Laura Nicolini. Milan, Il

Saggiatore (éd. orig. Cities in Evolution.

Londres, Williams \& Norgate).

- 1971 [1915]. Cities in Evolution, an Introduction to the Town Planning Movement and to the Study of Civics (édition de poche, introd. Pierre Clavel). Londres, Harper TorchbooksNew York, Dover.

- 1972 [1915]. "Cities in Evolution, an Introduction to the Town Planning Movement and to the Study of Civics ", in Marshall Stalley (éd.), Patrick Geddes : Spokesman for Man and the Environment. New Brunswick (NJ), Rutgers University Press : 105285.

- 1994a [1915]. Citades em evolução, par Maria José Ferreira de Castilho (d'après l'édition de 1949). Campinas, S. P. (Brésil), Papirus (Officio de Arte e Forma) (éd. orig. Cities in Evolution. Londres, Williams \& Norgate). - 1994b [1915]. L'évolution des villes, une introduction au mouvement de l'urbanisme et à l'étude de l'instruction civique, par Brigitte Ayramdjan. Paris, Temenos (éd. orig. Cities in Evolution. Londres, Williams \& Norgate). - 1996 [1915]. «City Survey for Town Planning Purposes, of Municipalities and Government» (chap. XVI de Cities in Evolution), in Richard T. Legates et Frederic Stout (éd.), The city reader. Londres-New York, Routledge-Thoemmes: 360-366.

- 1997 [1915]. Cities in Evolution, an Introduction to the Town Planning Movement and to the Study of Civics. Londres-New York, Routledge-Thoemmes Press, coll. «Social theories of the City", vol. 5.

- 1998 [1915]. Cities in Evolution, an Introduction to the Town Planning Movement and to the Study of Civics. Londres-New York, Routledge-Thoemmes Press, coll. «Early urban planning: 1870-1940», vol. 4. 
u GrIEvE, Robert. s. d. [1991]. Grieve on Geddes. Édimbourg, The Sir Patrick Geddes Memorial Trust.

u Hughes, Michael. 1971. The Letters of Lewis Mumford and Frederic J. Osborn: a Transatlantic Dialogue, 1938-70, LondresNew York, Praeger Publishers.

u Kitchen, Paddy. 1975. A Most Unsettling Person: The Life and Ideas of Patrick Geddes, Founding Father of City Planning and Environmentalism. New York, Saturday Review Press-E. P. Dutton \& Co.

u LANCHESTER, Henry Vaughan. 1932.

"Town Planning in India", The Sociological Review, vol. 24, n 4: 370-371.

u MAIRET, Philip. 1957. Pioneer of Sociology: the Life and Letters of Patrick Geddes. Londres, Lund Humphries.

u Meller, Hellen Elizatbeth. 1990. Patrick Geddes. Social evolutionist and city planner.

Londres, Routledge.

u MUMFoRD, Lewis. 1944. "Introduction», in Philip Boardman, Patrick Geddes: Maker of the Future. Chapel Hill, University of North Carolina Press: vii-xiv.

- 1950. «Mumford on Geddes», The Architectural Review, n 644: 80-84.

u NovaK, Frank G. (éd.). 1995. Lewis Mumford \& Patrick Geddes: the
Correspondance. Londres-New York, Routledge.

u PePler, George Lionel. 1932. «The Regional Planner», The Sociological Review, vol. $24, n^{\circ} 4: 382$.

u Sir Patrick Geddes Centenary Celebrations. Report of a Symposium held in the Edinburgh College of Art on Friday, $1^{\text {st }}$ October 1954. 1954. Édimbourg.

u STalley, Marshall (éd.). 1972. Patrick Geddes: Spokesman for Man and Environment, New Brunswick(NJ), Rutgers University Press.

u Sutcliffe, Anthony. 1994. «Naissance d'une discipline", in Jean Dethier et Alain Giheux (éd.). La Ville: art et architecture en Europe, 1870-1993. Paris, Centre Georges Pompidou: 121-128.

u TyrwhitT, Jaqueline Mary (éd.). 1947. Patrick Geddes in India (introd. L. Mumford, préf. H. V. Lanchester). Londres, Humphries.

- 1949. «Introduction», in Patrick Geddes, Cities in Evolution. Londres, Williams \& Norgate: ix-xxviii.

u Welter, Volker M. 2002. Biopolis: Patrick Geddes and the City of Life. Cambridge (MA)/Londres, The MIT Press. 